\title{
Adaptive Force/Velocity Control for Multi-Robot Cooperative Manipulation under Uncertain Kinematic Parameters
}

\author{
Sebastian Erhart and Sandra Hirche
}

\begin{abstract}
Multi-robot cooperative manipulation of a common object requires precise kinematic coordination of the attached end effectors in order to avoid excessive forces on the object and the manipulators. A manipulation task is considered successful if the desired object motion and forces are tracked accurately. In this paper we present a systematic analysis on the effect of uncertain kinematic parameters on the tracking behavior in a planar manipulation task. An adaptive control scheme is proposed, which achieves the desired control goal asymptotically. The presented scheme employs the current force/motion data of the attached end effectors without relying on a common reference frame. The algorithm is applicable to common manipulator types with wrist-mounted force/torque sensors and implementable in real-time. The performance of the proposed control scheme is evaluated experimentally with two 7DoF manipulators who cooperatively manipulate an object of uncertain length.
\end{abstract}

\section{INTRODUCTION}

A variety of manipulation tasks demands the cooperation of several potentially heterogeneous robots, e.g. in order to provide different sensing and actuation capabilities in manipulation tasks. A successful manipulation task is characterized by tracking a desired object motion while applying a desired force by means of the involved end effectors to the object. This subject motivated an intensive research on cooperative control schemes during the last three decades. However, there is evidence that cooperative control schemes fail to succeed when kinematic uncertainties arise in the manipulation task. This is usually the case for inaccuracies in the actual grasp location/orientation, in the relative pose of the cooperating manipulators and imprecise kinematic information on the manipulated object. A potential manipulation task involving two cooperating robots is depicted in Fig. 1.

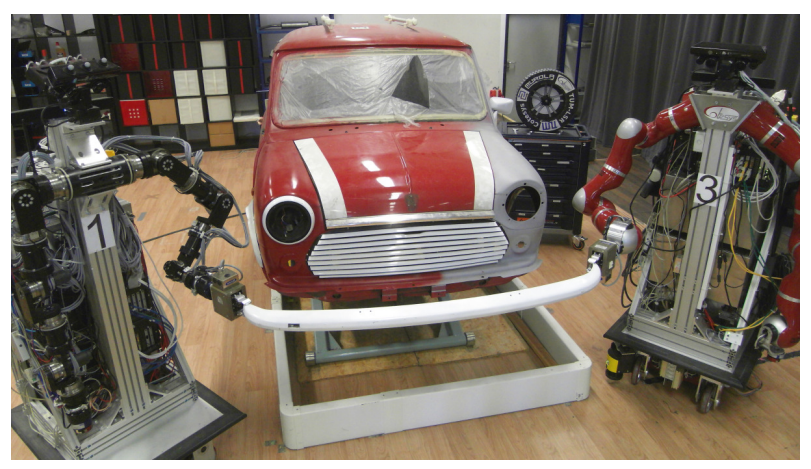

Fig. 1. Robots manipulating cooperatively a rigid object

S. Erhart and S. Hirche are with the Institute for Information-oriented Control, Technische Universtität München, 80290 München, Germany \{erhart, hirche\}@tum.de
The kinematic coordination is commonly achieved by using a common reference frame attached to the object. The kinematics of the individual manipulators are extended by a virtual stick [1] pointing to the origin of this reference frame. It is thus possible to compute the compatible end effector motion given the desired object motion in the reference frame. The ubiquitous presence of uncertainties in manipulation tasks requires the application of a force control scheme, among which impedance control [2] is probably the most widespread technique. This approach is used in [3] for a cooperative manipulation task to achieve compliance of the object in case of contact with the environment. The distributed impedance scheme in [4] guarantees asymptotic tracking of desired motion and force trajectories. In the presence of kinematic uncertainties, impedance-based control schemes offer a fair tradeoff between task performance and control complexity [5]. If the kinematic errors become large or objects have to be manipulated with a maximum level of position accuracy requiring a stiff control setting, common cooperative manipulation schemes should be extended by an adaptive controller.

Only few works [6]-[8] address the problem of kinematic uncertainties in the control design. In [6] an adaptive controller is presented dealing with uncertain kinematic parameters of a single robot. An adaptive control scheme for two cooperating manipulators with geometric uncertainties in the closed kinematic loop is presented in [7]. A least squares approach is used to identify the rigid transformation between the manipulators' end effector frames. While minimizing the actuator torques, the actual contact force is not addressed in the resulting control scheme. The work in [8] deals with the modeling and the control design for a single manipulator operating an uncertain kinematic mechanism. Although cooperative manipulators handling a common object are frequently subject to kinematic uncertainties, the consequences on position and force tracking are widely unexplored.

The contribution of this paper is an adaptive force/velocity control scheme for cooperative manipulation under uncertain kinematic parameters. As a first step we provide a thorough analysis of a planar, cooperative manipulation task. Based on a simple and comprehensible model we show that asymptotic force/velocity tracking and thus common control specifications for cooperative manipulation will generally not be met for uncertain kinematic parameters. We characterize the emerging interaction forces as a function of the kinematic uncertainties by deriving explicit formulas. We further present an adaptive control scheme for two manipulators and a planar manipulation task which is guaranteed to achieve the desired 
control performance. The presented algorithm is based on simple trigonometric computations and is thus suitable for real-time applications. The results are applicable to arbitrary manipulator types with wrist-mounted force/torque sensors.

The remainder of this paper is organized as follows. Section II is devoted to the modeling of the manipulation task and comprises the problem statement. Section III presents the adaptive controller which achieves the defined control goal in presence of uncertain kinematic parameters. The results of an experimental evaluation are illustrated in Section IV.

\section{MANIPULATION TASK MODEL}

In this paper we focus on a planar manipulation task involving $N$ independent manipulators as a prototypical case. Apart from the illustrative aspects of this simpler setting compared to the setting in the three-dimensional Euclidean group, a variety of manipulation tasks are either intrinsically planar, e.g. a transportation task, or can be approximated by such. Let the individual manipulator kinematics be characterized by a vector of joint angles $q_{i} \in \mathbb{R}^{N_{i}}$ and the manipulator Jacobian $J_{i}\left(q_{i}\right) \in \mathbb{R}^{2 \times N_{i}}$ for the $i$-th manipulator with $N_{i}$ degrees of freedom. The kinematics of a single manipulator, i.e. $q_{i}$ and $J_{i}$, are assumed to be known. Each manipulator is considered as velocity-controlled actuator according to

$$
\dot{q}_{i}=J^{+}\left(q_{i}\right) v_{i}^{r},
$$

given an individual reference velocity $v_{i}^{r} \in \mathbb{R}^{2}$ and the pseudo-inverse $J^{+}\left(q_{i}\right)$ for the $i$-th manipulator. The reference velocity

$$
v_{i}^{r}=v_{i}^{d}\left(\dot{x}_{o}^{d}\right)+v_{i}^{f}\left(\Delta f_{i}\right)
$$

comprises two elements. The first element $v_{i}^{d}$ is a feedforward velocity incorporating the desired object velocity

$$
\dot{x}_{o}^{d}=\left[\begin{array}{c}
\vec{v}_{o}^{d} \\
\dot{\varphi}_{o}^{d}
\end{array}\right],
$$

resulting from a trajectory planner, a superposed visual servo control loop or similar sources. The components $\vec{v}_{o}^{d} \in \mathbb{R}^{2}$ and $\dot{\varphi}_{o}^{d} \in \mathbb{R}$ denote the desired translational velocity and the desired angular velocity of the object respectively. The second component $v_{i}^{f}$ in (2) arises from a force feedback law controlling the individual force error $\Delta f_{i} \in \mathbb{R}^{2}$.

All end effectors are assumed to be tightly attached to a rigid object by means of a revolute joint. Consequently only the relative position of the manipulators is constrained through the object. Let $p_{i} \in \mathbb{R}^{2}$ refer to the current end effector position of the $i$-th manipulator, then the constraint reads as

$$
\left\|p_{j}-p_{i}\right\|^{2}=d_{j i}^{2}
$$

wherein $d_{j i} \in \mathbb{R}^{+}$is the distance between the end effectors for $j \neq i$. Differentiation of (4) with respect to time yields

$$
\left(p_{j}-p_{i}\right)^{T} \cdot\left(v_{j}^{r}-v_{i}^{r}\right)=0 .
$$

This means that the relative motion of two arbitrary end effectors is constrained to the subspace orthogonal to their connecting line. Assume that the desired relative motion $\left(v_{j}^{d}-v_{i}^{d}\right)$ does not meet the above orthogonality condition. As a consequence end effector forces emerge which lead to force errors and propagate in terms of the local feedback signals $v_{i}^{f}$ and $v_{j}^{f}$ to satisfy ultimately the constraint (5). Since in this case the effective end effector velocities are not equal to the desired velocities, i.e. $v_{i}^{r}-v_{i}^{d}=v_{i}^{f} \neq 0$, the actual object motion $\dot{x}_{o}$ will in general not coincide with its desired value $\dot{x}_{o}^{d}$. The desired control goal for the manipulation task,

$$
\dot{x}_{o} \rightarrow \dot{x}_{o}^{d} \quad, \quad \Delta f_{i} \rightarrow 0
$$

for $t \rightarrow \infty$ and an arbitrary value of $\dot{x}_{o}^{d}$, is therefore only met if the commanded velocities $v_{i}^{d}$ satisfy the constraint (5) asymptotically. This observation suggests that the coordination model for computing $v_{i}^{d}$ incorporates an estimate of the relative end effector positions $\left(p_{j}-p_{i}\right)$ which ensures compliance to the orthogonality constraint. Achieving the control goal (6) is indeed a challenging task since equation (5) for $N$ manipulators implies a total of $\frac{N(N-1)}{2}$ constraints to be met simultaneously. This results in a mathematically involved description of the emerging end effector forces as a superposition of all constraint forces associated to (5). In order to facilitate the integration of uncertain kinematic parameters and to provide comprehensible results in terms of compact mathematical expressions, the subsequent analysis is carried out for $N=2$ manipulators.

\section{A. Coordinate frame convention}

The desired object motion $\dot{x}_{o}^{d}$ is represented in an orthogonal, body-fixed coordinate system denoted by $\{o\}$ in Fig. 2 .

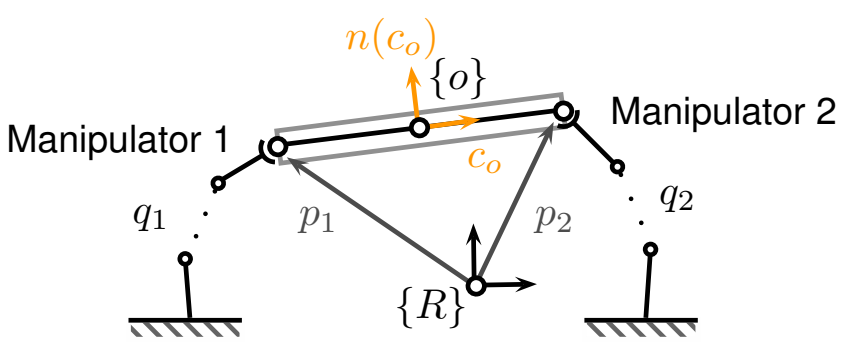

Fig. 2. Two manipulators carrying cooperatively a rigid object

The coordinate frame $\{o\}$ is assumed to be rigidly attached to the object such that its origin is located in the geometric center of the object and that its principal axis $c_{o}$ coincides with the connecting line of the end effectors, i.e.

$$
c_{o}=\frac{p_{2}-p_{1}}{\left\|p_{2}-p_{1}\right\|} .
$$

The vector $c_{o}$ defines not only the principal axis of the object frame but defines uniquely the orientation of the object in the plane with respect to a reference coordinate system $\{R\}$. The vector $c_{o}$ stands furthermore for the direction in which the motion of the end effectors is constrained when holding the object. That is the effective relative motion of 
the attached end effectors lies in the subspace orthogonal to the constrained direction. This subspace is spanned by the normal vector which results from rotating the original vector about 90 degrees in terms of the matrix operation $n(c)$ according to

$$
n(c)=\left[\begin{array}{cc}
0 & -1 \\
1 & 0
\end{array}\right] c
$$

With these definitions we determine the axis of the object frame as

$$
\{o\}=\left\{c_{o}, n\left(c_{o}\right)\right\}
$$

For the execution of the manipulation task, the desired object velocity $\dot{x}_{o}^{d}$ is transformed to compatible end effector velocities $v_{i}^{d}$ of the attached manipulators referring to the principle of virtual sticks [1]. The resulting transformation from the object frame $\{o\}$ to the origin of the respective end effector frame $\{i\}$ is subject to uncertainties. Each end effector uses consequently an estimate of the object frame $\hat{c}_{i}$ in its local coordinate frame $\{i\}$ for the desired transformation. This situation is depicted in Fig. 3.

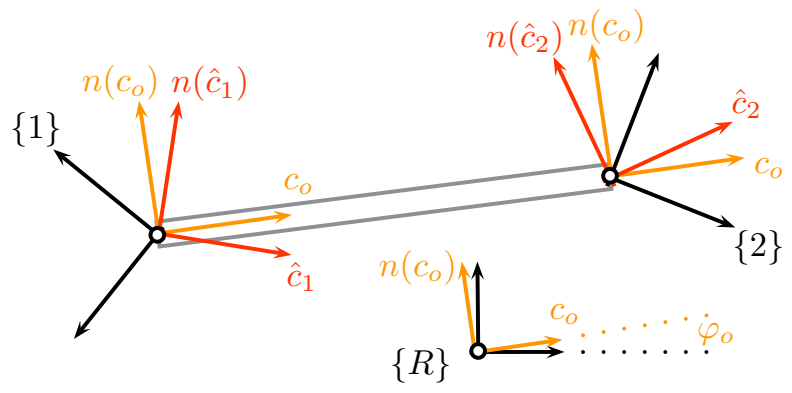

Fig. 3. End effector coordinate frames and estimates of the constraint

Apparently the estimates $\hat{c}_{i}$ are independent from each other and do not necessarily coincide with the actual, constrained direction $c_{o}$. For notational convenience we identify the unit vectors $c_{j}$ with the angles $\varphi_{j}$ formed with the principal axis of the reference frame $\{R\}$ (cf. Fig. 3) according to

$$
c_{j}\left(\varphi_{j}\right)=\left[\begin{array}{c}
\cos \varphi_{j} \\
\sin \varphi_{j}
\end{array}\right]
$$

for $j=\{o, 1,2\}$. By defining the angular errors

$$
\varepsilon_{i}=\varphi_{i}-\varphi_{o}
$$

for $i=\{1,2\}$ it is straightforward to verify

$$
\begin{aligned}
c_{o}^{T} \cdot \hat{c}_{i} & =\cos \varepsilon_{i} \\
c_{o}^{T} \cdot n\left(\hat{c}_{i}\right) & =-\sin \varepsilon_{i} .
\end{aligned}
$$

\section{B. Kinematic coordination model}

In order to simplify the kinematic coordination of the manipulators we assume that the object's motion consists solely of a translational component in the non-constrained direction $n\left(c_{o}\right)$ such that

$$
\vec{v}_{o}^{d}=v_{o}^{d} n\left(c_{o}\right)
$$

with $v_{o}^{d} \in \mathbb{R}$. The presented results straightforwardly extend to the case with a supplementary, translational velocity component in the direction of the constraint $c_{o}$. However this extension does not provide further insights and is omitted for the sake of compact mathematical expressions.

Given the individual estimates of the constrained direction $\hat{c}_{i}$, we may now transform the desired object velocity $\dot{x}_{o}^{d}$ into compatible end effector motions $v_{i}^{d}$. This is achieved by choosing

$$
\begin{aligned}
& v_{1}^{d}=n\left(\hat{c}_{1}\right) \cdot v_{o}^{d}-n\left(\hat{c}_{1}\right) \cdot \hat{v}_{\omega}^{d} \\
& v_{2}^{d}=n\left(\hat{c}_{2}\right) \cdot v_{o}^{d}+n\left(\hat{c}_{2}\right) \cdot \hat{v}_{\omega}^{d}
\end{aligned}
$$

wherein

$$
\hat{v}_{\omega}^{d}=\frac{1}{2} \hat{d} \dot{\varphi}_{o}^{d}
$$

denotes the translational end effector velocity due to a desired rotation. Note that in order to perform a proper rotation, the computation of $\hat{v}_{\omega}^{d}$ incorporates an estimate of the end effector distance $\hat{d} \in \mathbb{R}^{+}$. We further fix the center of rotation in the origin of the object frame which, for our particular choice of the object frame to lie in the geometrical center of the end effectors, results in minimal required kinetic end effector energy [9]. The signs for the terms involving $\hat{v}_{\omega}^{d}$ in (15) and (16) stem from the fact that the center of rotation is located in positive $\hat{c}_{1}$ and negative $\hat{c}_{2}$ direction.

Note further that the computation of the desired end effector velocities in (15) and (16) does not rely on the object frame $\{o\}$ but on the local estimates $\hat{c}_{i}$ of this frame. These local estimates are commonly represented in the corresponding end effector frame $\{i\}$. When rotating the object with a desired angular velocity $\dot{\varphi}_{o}^{d}$, it is thus selfevident to rotate the estimates $\hat{c}_{i}$ of the object frame, too. The vectorial description of this fact expressed in the reference frame $\{R\}$ (cf. Fig. 3) reads as

$$
\dot{\hat{c}}_{i}=n\left(\hat{c}_{i}\right) \dot{\varphi}_{o}^{d}
$$

for $i=\{1,2\}$. The same expression holds when rotating the individual end effector frame $\{i\}$ with $\dot{\varphi}_{o}^{d}$. In this case the estimate of the object frame $\hat{c}_{i}$, represented in the corresponding end effector frame, is constant.

\section{Force control model}

Each manipulator is endowed with a force control scheme in order to limit emerging forces and prevent damage of the object during the manipulation task. We consider the individual force feedback loop as part of the manipulator 
model justified by the strong relation between the kinematic coordination and the emerging forces. We restrict our analysis to the particular case of a proportional control given by

$$
v_{i}^{f}=\hat{c}_{i} \cdot k_{p, i} \cdot \Delta \hat{f}_{i}
$$

where $\Delta \hat{f}_{i} \in \mathbb{R}$ describes the force error of the $i$-th manipulator. Note that conventional implementations of impedance control use a second order filter to generate the desired motion signal $v_{i}^{f}$ from the force error $\Delta \hat{f}_{i}$. In general there is no immediate relation of the incorporated impedance tuning parameters denoted by mass, damping and stiffness to the parameters of a conventional PID controller. However the proportional control gain in (19) might be thought of as a non-zero damping with value $k_{p, i}^{-1}$ if mass and stiffness equal zero.

The error $\Delta \hat{f}_{i}$ is computed by projecting the measured force values $f_{i} \in \mathbb{R}^{2}$ onto the estimated constraint direction [8] and comparing those to a desired value $f^{d} \in \mathbb{R}$ according to

$$
\begin{aligned}
& \Delta \hat{f}_{1}=\hat{c}_{1}^{T} \cdot f_{1}-f^{d} \\
& \Delta \hat{f}_{2}=\hat{c}_{2}^{T} \cdot f_{2}+f^{d}
\end{aligned}
$$

In view of a consistent set point, the desired force values for the manipulators cannot be chosen independently. The measured force vectors $f_{1}$ and $f_{2}$ are assumed to have the same magnitude $f \in \mathbb{R}$ but opposed signs. The direction is imposed by the object orientation and reads thus as

$$
f_{1}=-f_{2}=-f c_{o}
$$

Consequently $f_{1}$ and $f_{2}$ represent the internal force components applied to the object. In analogy with rod mechanics, a positive force magnitude $f>0$ is assigned when pulling the object and a negative magnitude $f<0$ occurs when pushing against the object. Given the individual estimates $\hat{c}_{i}$ of the object frame it is possible to compute an estimate of gravitational and other external forces acting on the object. However, this estimate will in general be biased if $\hat{c}_{i} \neq c_{o}$.

\section{Kinematic uncertainty}

Biased estimates of the kinematic parameters will lead to undesired stress on the object and prevent accurate tracking of the desired object motion during the manipulation task. Both aspects will be detailed in the following.

1) Force analysis: In mechanical systems forces arise notably whenever a constraint tends to be violated. When there is no compliance, emerging forces become arbitrarily large in order to satisfy the kinematic constraint. In our manipulation task active compliance is provided to the manipulators in terms of a force feedback controller. We will derive an explicit expression for the occurring force as a function of the control parameters and the kinematic errors. To this end we employ (7) to rewrite the constraint equation (5) as

$$
c_{o}^{T} \cdot\left(v_{2}^{r}-v_{1}^{r}\right)=0
$$

We recall that $v_{i}^{r}$ depends on the force control $v_{i}^{f}$ by (2) and is consequently a function of the measured end effector force $f_{i}$ as stated in (19). Arbitrary values for the desired end effector velocities $v_{i}^{d}$ are a priori admissible. Nevertheless the emerging force magnitude $f$ will always adopt a suitable value such that the effective end effector velocities $v_{i}^{r}$ are a posteriori compliant to the constraint (23). In this perspective, the emerging end effector force can also be interpreted as the Lagrange multiplier for the system in (2) associated to the constraint (5). More precisely the resulting end effector force is the sum of all inter-manipulator forces necessary to respect the kinematic constraints. In case of two manipulators coupled through a single constraint the resulting end effector force is equal to the interaction force $f$ along the connecting line of the two end effectors.

We may explicitly compute its value for any constant $f^{d}$ by substituting equations (15), (16), (19), (20), (21) and (22) into (23). Letting $f^{d}=0$ and solving for $f$ yields

$$
f=-\frac{c_{o}^{T}\left[n\left(\hat{c}_{2}\right)+n\left(\hat{c}_{1}\right)\right] \cdot \hat{v}_{\omega}^{d}+c_{o}^{T}\left[n\left(\hat{c}_{2}\right)-n\left(\hat{c}_{1}\right)\right] \cdot v_{o}^{d}}{c_{o}^{T}\left[k_{p, 2}\left(\hat{c}_{2}^{T} c_{o}\right) \hat{c}_{2}+k_{p, 1}\left(\hat{c}_{1}^{T} c_{o}\right) \hat{c}_{1}\right]}
$$

This equation provides the mathematical description for the following interesting insight. The vector difference $\left[n\left(\hat{c}_{2}\right)-n\left(\hat{c}_{1}\right)\right]$ is orthogonal to $c_{o}$ whenever the vectors $\hat{c}_{2}$ and $\hat{c}_{1}$ point in the same direction. Performing a pure translational motion $\left(v_{o}^{d} \neq 0, \hat{v}_{\omega}^{d}=0\right)$ we have $f=f^{d}$ when the estimates of the constrained direction are biased by the same magnitude

$$
\hat{c}_{1}=\hat{c}_{2}
$$

Less obvious is the fact that for a pure rotational motion $\left(\hat{v}_{\omega}^{d} \neq 0, v_{o}^{d}=0\right)$ we have $f=f^{d}$ when $\left[n\left(\hat{c}_{2}\right)+n\left(\hat{c}_{1}\right)\right]$ is perpendicular to $c_{o}$. That is the estimates are biased by the same magnitude but having opposed signs. This situation is sketched in Fig. 3 and reads mathematically as

$$
c_{o}^{T} \cdot \hat{c}_{1}=-c_{o}^{T} \cdot \hat{c}_{2} .
$$

In case of $\hat{v}_{\omega}^{d} \neq 0$ and $v_{o}^{d} \neq 0$ we have $f=f^{d}$ for

$$
c_{o}^{T} \cdot\left[n\left(\hat{c}_{2}\right)-n\left(\hat{c}_{1}\right)\right] v_{o}^{d}=-c_{o}^{T} \cdot\left[n\left(\hat{c}_{2}\right)+n\left(\hat{c}_{1}\right)\right] \hat{v}_{\omega}^{d}
$$

which holds particularly for

$$
\hat{c}_{1}=\hat{c}_{2}=c_{0} .
$$

This means the estimates of the constrained direction match perfectly which is rarely the case for some initial estimates.

Actually (27) admits a solution to $f=f^{d}$ different from (28). For particular values of $\hat{v}_{\omega}^{d} \neq 0$ and $v_{o}^{d} \neq 0$ the emerging force magnitude will coincide with the desired value even though the estimates $\hat{c}_{1}$ and $\hat{c}_{2}$ do not match $c_{o}$. This fact can be regarded as an excitation condition for forcebased parameter identification in cooperative manipulation tasks and is further addressed in Section III. 


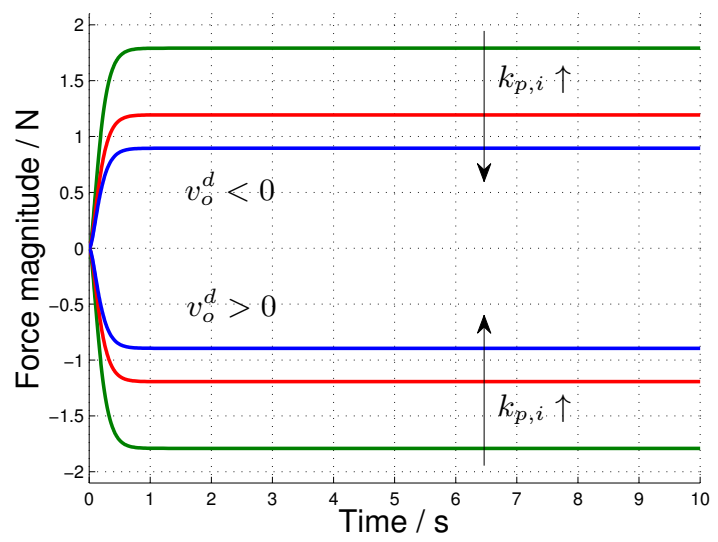

Fig. 4. Interaction force $f$ for a translational motion $v_{o}^{d}$ with biased estimates $\hat{c}_{i}$ and varying control parameters $k_{p, i}$

A numeric example is presented to illustrate the above findings. Consider again the configuration depicted in Fig. 3 wherein the angular errors are set to $\varepsilon_{1}=-10^{\circ} \frac{\pi \mathrm{rad}}{180^{\circ}}$ and $\varepsilon_{2}=+10^{\circ} \frac{\pi \mathrm{rad}}{180^{\circ}}$. The desired force value is set to $f^{d}=0$. The result of a numeric simulation for $v_{o}^{d}= \pm 0.05 \frac{\mathrm{m}}{\mathrm{s}}, \hat{v}_{\omega}^{d}=0$ and different values for $k_{p, i}=\{0.005,0.0075,0.010\} \frac{\mathrm{m}}{\mathrm{Ns}}$ is plotted in Fig. 4.

A positive translation $v_{o}^{d}>0$ squeezes the object $(f<0)$ while a negative translation $v_{o}^{d}<0$ causes the attached end effectors to pull the object $(f>0)$. Given the desired motion of the end effectors, the emerging interaction force $f$ causes the end effectors to respect the kinematic constraint regardless of the current angular errors $\varepsilon_{i}$. Interestingly the value of $f$ depends not only on the angular errors $\varepsilon_{i}$ but on the value of the control gains $k_{p, i}$, too. Increasing values for $k_{p, i}$ result in a decreasing force magnitude $f$. While the control gains $k_{p, i}$ in the presented simulation were chosen to be identical for all manipulators, the preceding observation allows for more sophisticated tuning strategies of the control parameters depending on the individual estimation accuracy of the parameters $\hat{c}_{i}$. Manipulators with inaccurate estimates $\hat{c}_{i}$ should tend to increase their control gains in order to limit the emerging force error.

2) Velocity analysis: Whenever undesired forces occur during the manipulation task, the effectively followed trajectory of the end effectors will diverge from their desired trajectory. We are now interested in the effective instantaneous velocity of the object. The translational velocity of the virtual object frame is the average of the end effector velocities projected onto the non-constrained direction

$$
v_{o}=n\left(c_{o}\right)^{T} \cdot\left(\frac{1}{2}\left[v_{1}^{r}+v_{2}^{r}\right]\right) .
$$

The object's angular velocity results from the relative motion of the end effectors projected onto the non-constrained direction

$$
\dot{\varphi}_{o}=\frac{n\left(c_{o}\right)^{T} \cdot\left(v_{2}^{r}-v_{1}^{r}\right)}{d} .
$$

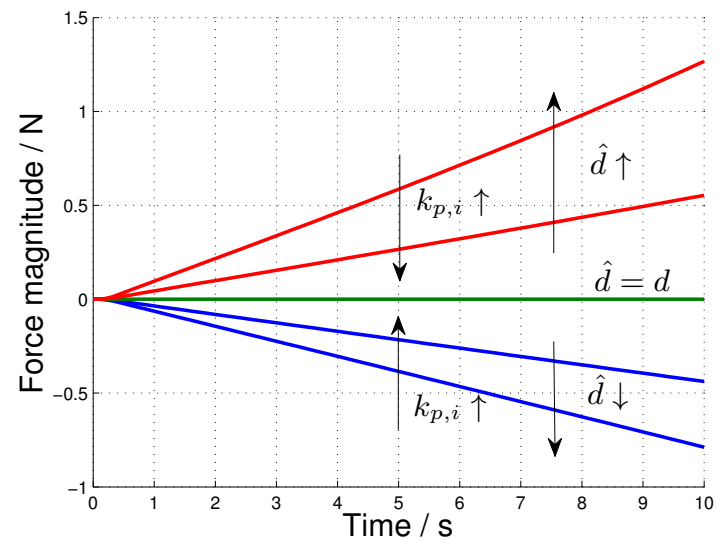

Fig. 5. Interaction force $f$ for a rotational motion $\dot{\varphi}_{o}^{d}$ with biased estimate $\hat{d}$

The actual end effector distance $d$ is required to compute the effective angular velocity of the object. Both velocities, $v_{o}$ and $\dot{\varphi}_{o}$, coincide with their desired values $v_{o}^{d}$ and $\dot{\varphi}_{o}^{d}$ only if condition (28) holds and the estimated end effector distance equals the actual one

$$
\hat{d}=d
$$

The significance of the parameter $\hat{d}$ is illustrated by means of an example. Assume that the initial estimates $\hat{c}_{i}$ are unbiased and the angular error is thus zero when starting the manipulation task, i.e. $\varepsilon_{i}(0)=0$. Let the end effector distance be $d=0.5 \mathrm{~m}$ and assume that the manipulators rotate the object with a desired angular velocity of $\dot{\varphi}_{o}^{d}=0.1 s^{-1}$ while $v_{o}^{d}=0$. The force controllers are tuned with $f^{d}=0$ and $k_{p, i}=0.005 \frac{\mathrm{m}}{\mathrm{Ns}}$. A numeric simulation is conducted for different values of the estimated end effector distance $\hat{d}=\{0.40,0.45,0.50,0.55,0.6\} \mathrm{m}$. The result is plotted in Fig. 5.

Clearly the interaction force of the manipulators diverges from its desired value $f^{d}=0$ for any estimate $\hat{d} \neq d$. A very descriptive explanation for the emerging interaction force in Fig. 5 is that for any $\hat{d} \neq d$ the resulting diameter of the circle described by the desired end effector motion $v_{i}^{d}$ is either too small or too big. As the actual end effector motion $v_{i}^{r}$ is forced to maintain the distance $d$, the manipulation task will either result in compressing the object for $\hat{d}<d$ or pulling the object for $\hat{d}>d$. The mathematical description of this observation reads as follows. For $\varepsilon_{i}=0$ it follows from (30) that $\dot{\varphi}_{o}=\frac{\hat{d}}{d} \dot{\varphi}_{o}^{d}$. That is the effective angular velocity of the object differs from its desired value by the ratio of estimated and actual end effector distance. Since the estimates of the object orientation $\hat{c}_{i}$ rotate with the desired angular velocity $\dot{\varphi}_{o}^{d} \neq \dot{\varphi}_{o}$ according to (18), the angular errors $\varepsilon_{i}$ will consequently increase which in turn leads to a force error according to (24). The desired force value is only maintained in case of $\varepsilon_{i}=0$ and $\hat{d}=d$ and motivates an adaptive controller which meets the desired control goal (6) under uncertain kinematic parameters. 


\section{ADAPTIVE CONTROLLER DESIGN}

For the design of the adaptive control law it is convenient to introduce the reciprocals of estimated and actual end effector distance

$$
\hat{\delta}=\frac{1}{\hat{d}} \quad, \quad \delta=\frac{1}{d} .
$$

With the definition above and use of equations (12) and (13) we rewrite (30) as

$$
\dot{\varphi}_{o}=\delta \psi\left(\varepsilon_{i}, \hat{\delta}, \dot{x}_{o}^{d}\right)
$$

with

$$
\begin{aligned}
\psi\left(\varepsilon_{i}, \hat{\delta}, \dot{x}_{o}^{d}\right)=\quad & {\left[\cos \varepsilon_{2}-\cos \varepsilon_{1}\right] v_{o}^{d} } \\
& +\left[\cos \varepsilon_{2}+\cos \varepsilon_{1}\right] \hat{v}_{\omega}^{d} \\
& +\xi\left(\varepsilon_{1}, \varepsilon_{2}\right)\left[\sin \varepsilon_{1}-\sin \varepsilon_{2}\right] v_{o}^{d} \\
& -\xi\left(\varepsilon_{1}, \varepsilon_{2}\right)\left[\sin \varepsilon_{1}+\sin \varepsilon_{2}\right] \hat{v}_{\omega}^{d}
\end{aligned}
$$

and

$$
\xi\left(\varepsilon_{1}, \varepsilon_{2}\right)=\frac{k_{p, 1} \sin \varepsilon_{1} \cos \varepsilon_{1}+k_{p, 2} \sin \varepsilon_{2} \cos \varepsilon_{2}}{k_{p, 2} \cos ^{2} \varepsilon_{2}+k_{p, 1} \cos ^{2} \varepsilon_{1}} .
$$

The kinematic coordination model of the manipulation task written in a compact form becomes

$$
\begin{aligned}
\dot{\varphi}_{1} & =\dot{\varphi}_{o}^{d}+u_{\varphi_{1}} \\
\dot{\varphi}_{2} & =\dot{\varphi}_{o}^{d}+u_{\varphi_{2}} \\
\dot{\varphi}_{o} & =\delta \psi\left(\varepsilon_{i}, \hat{\delta}, \dot{x}_{o}^{d}\right) \\
\dot{\hat{\delta}} & =u_{\delta}
\end{aligned}
$$

where the additional control inputs $u_{\varphi_{1}}, u_{\varphi_{2}}$ and $u_{\delta}$ have been introduced in order to update the estimate of the kinematic parameters. The design of adaptive controllers for this class of systems is e.g. addressed in [10].

Proposition 1: The control law

$$
\begin{aligned}
u_{\varphi_{1}} & =-\dot{\varphi}_{o}^{d}+\hat{\delta} \psi\left(\varepsilon_{i}, \hat{\delta}, \dot{x}_{o}^{d}\right)-k_{\varepsilon_{1}} \varepsilon_{1} \\
u_{\varphi_{2}} & =-\dot{\varphi}_{o}^{d}+\hat{\delta} \psi\left(\varepsilon_{i}, \hat{\delta}, \dot{x}_{o}^{d}\right)-k_{\varepsilon_{2}} \varepsilon_{2} \\
u_{\delta} & =-k_{\delta}\left(\varepsilon_{1}+\varepsilon_{2}\right) \psi\left(\varepsilon_{i}, \hat{\delta}, \dot{x}_{o}^{d}\right)
\end{aligned}
$$

stabilizes the system (36) asymptotically in the sense of Lyapunov about the equilibrium point

$$
z_{0}=\left[\begin{array}{c}
\varepsilon_{1} \\
\varepsilon_{2} \\
\hat{\delta}
\end{array}\right]=\left[\begin{array}{l}
0 \\
0 \\
\delta
\end{array}\right]
$$

for $k_{\varepsilon_{1}}, k_{\varepsilon_{2}}>0$ and $k_{\delta}=1$. Furthermore, the controller achieves asymptotic tracking of the desired object velocity $\dot{x}_{o}^{d}$ and the desired end effector force $f^{d}$ for $\dot{\varphi}_{o}^{d} \neq 0$ and $\left\|\dot{x}_{o}^{d}\right\|<\infty$.

\section{Proof:}

Straightforward computation of the error dynamics and substituting $u_{\varphi_{1}}, u_{\varphi_{2}}$ and $u_{\delta}$ from (37) yields

$$
\begin{aligned}
\dot{\varepsilon}_{1} & =[\hat{\delta}-\delta] \psi\left(\varepsilon_{i}, \hat{\delta}, \dot{x}_{o}^{d}\right)-k_{\varepsilon_{1}} \varepsilon_{1} \\
\dot{\varepsilon}_{2} & =[\hat{\delta}-\delta] \psi\left(\varepsilon_{i}, \hat{\delta}, \dot{x}_{o}^{d}\right)-k_{\varepsilon_{2}} \varepsilon_{2} \\
\dot{\hat{\delta}} & =-k_{\delta}\left(\varepsilon_{1}+\varepsilon_{2}\right) \psi\left(\varepsilon_{i}, \hat{\delta}, \dot{x}_{o}^{d}\right) .
\end{aligned}
$$

Consider the Lyapunov candidate

$$
V\left(\varepsilon_{1}, \varepsilon_{2}, \hat{\delta}\right)=\frac{1}{2}\left(\varepsilon_{1}^{2}+\varepsilon_{2}^{2}+[\hat{\delta}-\delta]^{2}\right) .
$$

The time derivative is

$$
\begin{aligned}
\dot{V}\left(\varepsilon_{1}, \varepsilon_{2}, \hat{\delta}\right)= & \varepsilon_{1} \dot{\varepsilon}_{1}+\varepsilon_{2} \dot{\varepsilon}_{2}+[\hat{\delta}-\delta] u_{\delta} \\
= & \varepsilon_{1}\left([\hat{\delta}-\delta] \psi\left(\varepsilon_{i}, \hat{\delta}, \dot{x}_{o}^{d}\right)-k_{\varepsilon_{1}} \varepsilon_{1}\right) \\
& +\varepsilon_{2}\left([\hat{\delta}-\delta] \psi\left(\varepsilon_{i}, \hat{\delta}, \dot{x}_{o}^{d}\right)-k_{\varepsilon_{2}} \varepsilon_{2}\right) \\
& -[\hat{\delta}-\delta]\left(\varepsilon_{1}+\varepsilon_{2}\right) \psi\left(\varepsilon_{i}, \hat{\delta}, \dot{x}_{o}^{d}\right) \\
= & -k_{\varepsilon_{1}} \varepsilon_{1}^{2}-k_{\varepsilon_{2}} \varepsilon_{2}^{2} \\
\leq & 0
\end{aligned}
$$

In order to conclude on the convergence of $\varepsilon_{1}$ and $\varepsilon_{2}$ one considers

$$
\ddot{V}\left(\varepsilon_{1}, \varepsilon_{2}, \hat{\delta}\right)=-2 k_{\varepsilon_{1}} \varepsilon_{1} \dot{\varepsilon}_{1}-2 k_{\varepsilon_{2}} \varepsilon_{2} \dot{\varepsilon}_{2}
$$

which is bounded on a domain containing $z_{0}$ and given a bounded $\dot{x}_{o}^{d}$. Barbalat's Lemma implies $\dot{V} \rightarrow 0$ and thus $\varepsilon_{i} \rightarrow 0$ as $t \rightarrow \infty$. Employing this fact for $\dot{\varepsilon}_{i}$ in (39) for $t \rightarrow \infty$ leads to $\left(1-\frac{\delta}{\hat{\delta}}\right) \dot{\varphi}_{o}^{d}=0$, which can only hold true if $\hat{\delta} \rightarrow \delta$ for $\dot{\varphi}_{o}^{d} \neq 0$. Convergence of the error dynamics to the equilibrium point $z_{0}$ implies $\Delta f_{i} \rightarrow 0$ by means of equation (24) and further $\dot{x}_{o} \rightarrow \dot{x}_{o}^{d}$ in terms of equations (29) and (30).

A few remarks which further characterize the presented result are in place:

- Asymptotic convergence of the control law (37) relies implicitly on the boundedness of $\xi\left(\varepsilon_{1}, \varepsilon_{2}\right)$ in (35). However this term is infinite for $\varepsilon_{i}= \pm \frac{\pi}{2}$. By choosing initial values

$$
\varepsilon_{1}^{2}(0)+\varepsilon_{2}^{2}(0)+[\hat{\delta}(0)-\delta]^{2}<\left(\frac{\pi}{2}\right)^{2}
$$

sufficiently close to the equilibrium point $z_{0}$ one can guarantee the boundedness for $t \geq 0$ by some invariancelike property [11]. This indicates the local character of the stability proof.

- Application of the control law demands the computation of the angular errors $\varepsilon_{i}$. Given the constraint estimates $\hat{c}_{i}$ in the particular end effector frame (cf. Fig. 3), each manipulator infers its individual angular error from the measured end effector force $f_{i}$. According to (22) the end effector forces are collinear to the actual constraint direction $c_{o}$ such that the desired computation of $\varepsilon_{i}$ is achieved via

$$
\varepsilon_{i}=\operatorname{atan} 2\left(\hat{c}_{i}^{2}, \hat{c}_{i}^{1}\right)-\operatorname{atan} 2\left(\sigma_{i} \cdot f_{i}^{2}, \sigma_{i} \cdot f_{i}^{1}\right)
$$


wherein the upper index denotes the projection of the vector to the first and second axis of the local end effector frame respectively. The function $\sigma_{i}=\operatorname{sgn}\left(\hat{c}_{i}^{T} f_{i}\right)$ in (44) takes care of computing always the smallest angle between $\hat{c}_{i}$ and the line defined by $f_{i}$. The computation of $\varepsilon_{i}$ is well-defined for non-zero end effector forces $\left\|f_{i}\right\| \neq 0$ which can be enforced by letting $f^{d} \neq 0$. A particular situation arises for $f^{d}=0$. A concise conclusion on $\varepsilon_{i}=0$ from the observation $\left\|f_{i}\right\|=0$ is only admissible if the additional excitation condition

$$
\tan \left(\frac{\varepsilon_{1}-\varepsilon_{2}}{2}\right) v_{o}^{d} \neq \tan \left(\frac{\varepsilon_{1}+\varepsilon_{2}}{2}\right) \hat{v}_{\omega}^{d}
$$

is met. This condition stems from equation (27) which characterizes the null-space of a desired object motion given the measured end effector forces as the output. Furthermore it should be noted that the angular error computation as proposed in (44) is increasingly sensitive to potential sensor noise as $\left\|f_{i}\right\|$ tends to zero.

\section{EXPERIMENTAL EVALUATION}

An experimental study of the presented control scheme is conducted involving two anthropomorphic manipulators with $N_{i}=7$ degrees of freedom. The low-level control scheme of each manipulator is a joint-space position controller which provides an interface to track a Cartesian reference velocity similar to (1). The control loops are closed with a sampling rate of $T_{s}=1 \mathrm{~ms}$. The manipulators are equipped with a wrist-mounted JR3 67M25 6-dimensional force/torque sensor and a Schunk PG70 end effector. Both end effectors are rigidly connected to an aluminum bar of length $d=0.485 \mathrm{~m}$ via revolute joints. The experiment is conducted by means of a dual-arm manipulator depicted in Fig. 6.

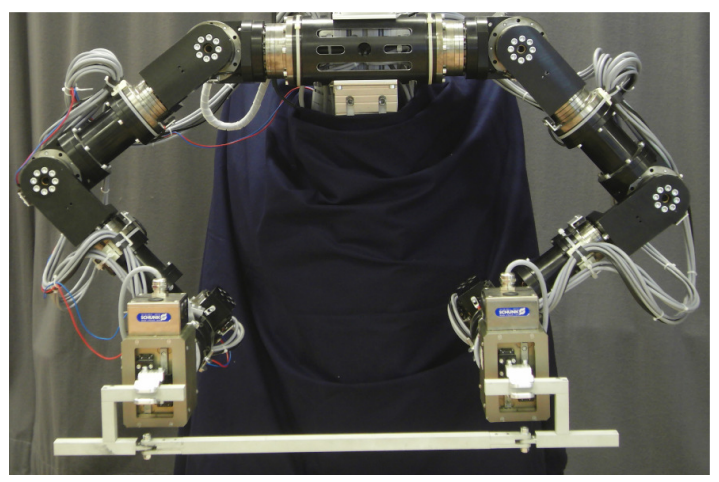

Fig. 6. Experimental setup with two 7DoF manipulators

No common reference frame is used in order to guarantee the effectiveness of the approach for the case of two completely independent manipulators as depicted in Fig. 1. The force measurements are filtered by a low-pass filter with cutoff frequency $f_{c}=125 \mathrm{~Hz}$. The desired force value is set to $f^{d}=-5 \mathrm{~N}$. The force control gains are $k_{p, i}=0.001 \frac{\mathrm{m}}{\mathrm{Ns}}$. The control gains relevant to the angular errors are set to $k_{\varepsilon_{i}}=0.5 \mathrm{~s}^{-1}$. A first experiment is conducted in order to illustrate the performance of the presented computations. To this end the estimates of the constrained direction $\hat{c}_{i}$ are intentionally biased resulting in $\varepsilon_{1}(0)=30^{\circ} \frac{\pi \mathrm{rad}}{180^{\circ}}$ and $\varepsilon_{2}(0)=-15^{\circ} \frac{\pi \mathrm{rad}}{180^{\circ}}$. These values may result from an ordinary visual servo control routine for the end effector positioning assuming a conservative error bound. While keeping the manipulators at rest, i.e. $\dot{x}_{o}^{d}=0$, the adaptive controller is enabled at time instant $t=1 \mathrm{~s}$. Note that the choice of $f^{d} \neq 0$ enables the computation of the angular errors even without any object motion. The graph of the resulting experimental angular errors is depicted in Fig. 7.

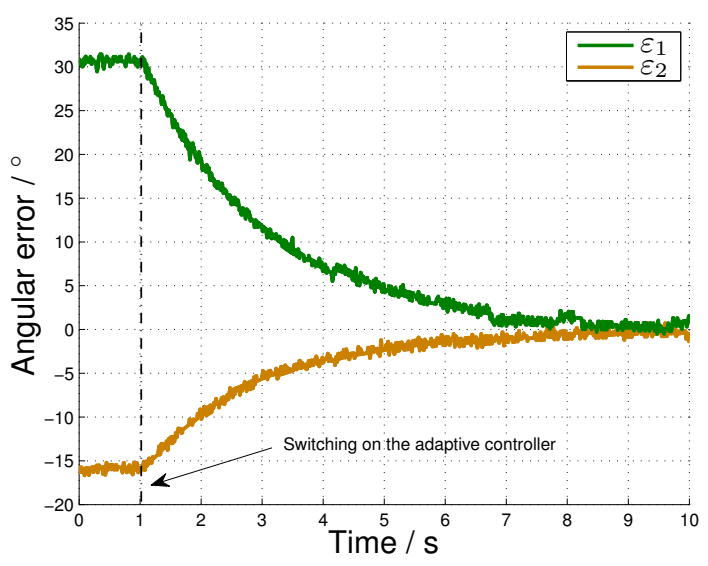

Fig. 7. Convergence of the angular errors to zero after switching on the adaptive controller at $t=1 \mathrm{~s}$

As expected both angular errors $\varepsilon_{i}$ converge perfectly to zero. For a non-zero angular velocity $\dot{\varphi}_{o}^{d}$ convergence of $\varepsilon_{i}$ to zero occurs only if $\hat{\delta}=\delta$. This will be studied in the following assuming small initial angular errors $\varepsilon_{i}(0) \approx 0$.

Due to the workspace constraints of the manipulators, a periodic signal with zero mean is chosen for the desired angular velocity of the object according to $\dot{\varphi}_{o}^{d}(t)=\hat{\dot{\varphi}} \sin \left(\omega_{\dot{\varphi}} t\right)$ with $\hat{\dot{\varphi}}=0.06 s^{-1}$ and $\omega_{\dot{\varphi}}=\frac{2 \pi}{20} s^{-1}$. The translational velocity is kept at $v_{o}^{d}=0$. The remaining parameter of the adaptive controller is set to $k_{\delta}=33$. The choice of $k_{\delta}>1$ turned out to increase the convergence speed for the parameter $\hat{\delta}$ in the experiments significantly. According to $\dot{\hat{\delta}}$ in equation (39), faster convergence of $\hat{\delta}$ is expected for a growing magnitude of $\dot{\varphi}_{o}^{d}$, too.

The estimate of the end effector distance $\hat{d}$ is plotted for two distinct runs with different initial values. The result of a first run with $\hat{d}(0)=0.7 \mathrm{~m}$ is depicted along the result of a numeric simulation with identical parameters in Fig. 8.

The convergence of $\hat{d}$ from the experimental data is clearly visible. Nevertheless the convergence speed expected from the simulation is not reached. This may have several reasons. A delay between commanded and performed motion in combination with a perceptible backlash of manipulators and object might not only cause an amplification of the periodic bumps. It causes simultaneously the convergence rate to differ from its prediction.

A second run is performed with an initial estimate of $\hat{d}(0)=0.2 \mathrm{~m}$. The results are illustrated in Fig. 9 .

Again we observe convergence of the estimated end ef- 


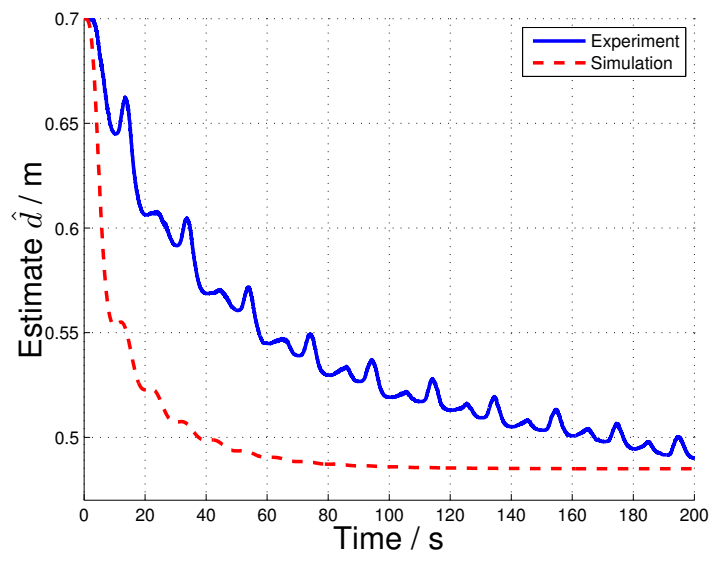

Fig. 8. Estimated end effector distance for $\hat{d}(0)=0.7 \mathrm{~m}$

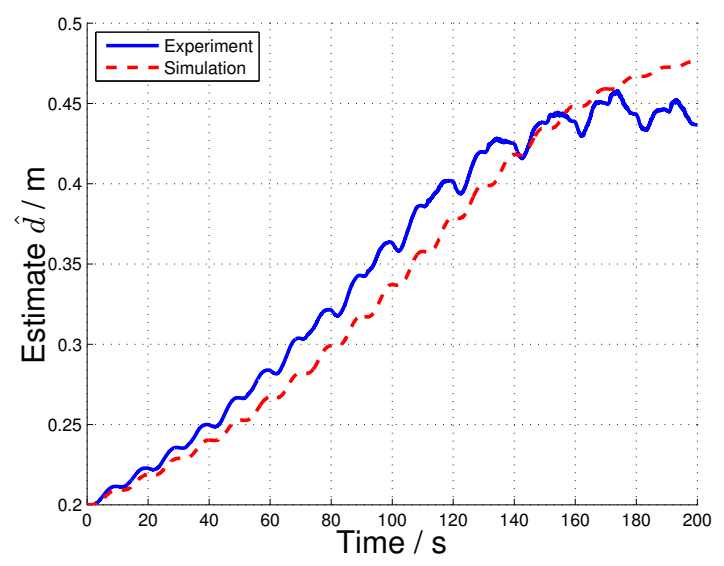

Fig. 9. Estimated end effector distance for $\hat{d}(0)=0.2 m$

fector distance. This time the convergence rate is slightly faster than predicted by the numeric simulation. However, the estimate tends to $\hat{d} \approx 0.45 \mathrm{~m}$ and the actual value $d=0.485 \mathrm{~m}$ is not reached. This observation is mainly attributed to inaccuracies in the kinematic calibration of the manipulator. Moreover, mechanical oscillations due to the Harmonic Drive gears in use for the manipulators counteracted the usability of the force data and prevented thus an experimental evaluation for increased end effector velocities.

\section{A. Discussion}

We observed in the experiments that condition (43), describing an invariant sublevel set for $V$ in (40), might be conservative. While the initial value $\hat{d}(0)=0.7 \mathrm{~m}$ is compliant to condition (43), the convergence for $\hat{d}(0)=0.2 \mathrm{~m}$ is not covered by the presented criterion. There might be situations for $\varepsilon_{i}$ close to $\pm \frac{\pi}{2}$ where $\hat{\delta} \neq \delta$ and a sufficiently large $\dot{\varphi}_{o}^{d}$ leads to $\varepsilon_{i}= \pm \frac{\pi}{2}$. Less conservative sets are supposed to be found by taking into account the actual values of the control gains $k_{\varepsilon_{i}}$ and further assumptions on the desired velocity $\dot{\varphi}_{o}^{d}$.

The convergence rate for $\hat{\delta}$ is shown to depend on the value of the desired velocity $\dot{\varphi}_{o}^{d}$ by means of equation (39).
However, the choice of the parameters $k_{\varepsilon_{i}}$ has an impact on the convergence of $\hat{\delta}$, too. Large values for $k_{\varepsilon_{i}}$ will keep the angular errors $\varepsilon_{i}$ small and lead consequently to a small $\dot{\hat{\delta}}$ in equation (39). This observation further motivates the selection of $k_{\delta}>1$ for an increased convergence rate of $\hat{\delta}$ during the experiments. A stability proof for increased values of $k_{\delta}$ is the subject of ongoing research.

\section{CONCLUSION}

In this paper a planar, cooperative manipulation task subject to uncertain kinematic parameters is investigated. A systematic analysis of force and velocity signals reveals the disturbing impact of kinematic uncertainties on the desired control goal in terms of position and force tracking. We present an adaptive controller which achieves provably the postulated control goal for uncertain kinematic parameters and provide experimental results which confirm the relevance and the applicability of our findings. Future research will include the analysis of additional disturbances to the system and the transfer to a general, three-dimensional manipulation task.

\section{ACKNOWLEDGMENT}

This research is partly supported by the DFG excellence initiative research cluster "CoTeSys - Cognition for Technical Systems" and the European Union Seventh Framework Programme FP7/2007-2013 under grant agreement no. 601165 of the project "WEARHAP - Wearable Haptics for Humans and Robots".

\section{REFERENCES}

[1] B. Siciliano and O. Khatib, Springer Handbook of Robotics. Secaucus, NJ, USA: Springer-Verlag New York, Inc., 2007.

[2] N. Hogan, "Impedance Control: An Approach to Manipulation: Part I - Theory," Journal of Dynamic Systems, Measurement, and Control, vol. 107, no. 1, pp. 1-7, 1985.

[3] S. Schneider and J. Cannon, R.H., "Object impedance control for cooperative manipulation: theory and experimental results," Robotics and Automation, IEEE Transactions on, vol. 8, pp. 383 -394, jun 1992

[4] J. Szewczyk, F. Plumet, and P. Bidaud, "Planning and controlling cooperating robots through distributed impedance," Journal of Robotic Systems, vol. 19, no. 6, pp. 283-297, 2002.

[5] S. Erhart, D. Sieber, and S. Hirche, "An impedance-based control architecture for multi-robot cooperative dual-arm mobile manipulation," in Intelligent Robots and Systems (IROS), 2013 IEEE/RSJ International Conference on, Nov. 2013.

[6] C. Cheah, C. Liu, and J. Slotine, "Adaptive Jacobian tracking control of robots with uncertainties in kinematic, dynamic and actuator models," Automatic Control, IEEE Transactions on, vol. 51, pp. 1024 - 1029, June 2006.

[7] F. Aghili, "Self-tuning cooperative control of manipulators with position/orientation uncertainties in the closed-kinematic loop," in Intelligent Robots and Systems (IROS), 2011 IEEE/RSJ International Conference on, pp. $4187-4193$, sept. 2011.

[8] Y. Karayiannidis, C. Smith, F. Via, P. Ogren, and D. Kragic, "Open sesame! Adaptive force/velocity control for opening unknown doors," in Intelligent Robots and Systems (IROS), 2012 IEEE/RSJ International Conference on, pp. 4040-4047, Oct.

[9] J. Markdahl, Y. Karayiannidis, X. Hu, and D. Kragic, "Distributed cooperative object attitude manipulation," in Robotics and Automation (ICRA), 2012 IEEE International Conference on, pp. 2960-2965, May.

[10] R. Ortega, A. Astolfi, and N. Barabanov, "Nonlinear PI control of uncertain systems: an alternative to parameter adaptation," vol. 47, pp. 259-278, 2002.

[11] H. K. Khalil, Nonlinear Systems. Englewood Cliffs, NJ, USA: Prentice-Hall, Inc., 2002. 